\title{
Incidencia e importancia pronóstica del deterioro de la función renal en pacientes hospitalizados con insuficiencia cardiaca
}

\author{
PABLO CASTRO ${ }^{1}$, HUGO VERDEJO ${ }^{1}$, RODRIGO ALTAMIRANO', \\ PATRICIO DOWNEY ${ }^{1}$, JOSÉ LUIS VUKASOVIC ${ }^{2}$, LUIS SEPÚLVEDA ${ }^{3}$, \\ FERNANDO LANAS ${ }^{4}$, PAOLA BILBAO ${ }^{3}$, RICARDO PACHECO ${ }^{3}$, \\ VÍCTOR ROSSEL ${ }^{2}$ EN REPRESENTACIÓN GRUPO ICARO
}

\section{Deterioration of kidney function as a risk factor for mortality among patients hospitalized for heart failure}

Background: Acute deterioration of kidney function among patients admitted to the hospital for cardiac failure is associated with an increased mortality. Aim: To investigate the association between deterioration of kidney function and mortality among patients hospitalized for cardiac failure. Material and Methods: Patients admitted for decompensated cardiac failure to 14 Chilean hospitals between 2002 and 2009 were incorporated to the study. Clinical and laboratory features were registered. Serum creatinine values on admission and discharge were determined. Hospital and long term mortality was determined requesting death certificates to the National Identification Service at the end of follow up, lasting $635 \pm 581$ days. Results: One thousand sixty four patients were incorporated and 1100, aged $68 \pm 13$ years (45\% females) had information about renal function. Seventy seven percent were hypertensive and $36 \%$ were diabetic. Mean ejection fraction was $41 \pm 18 \%$ and $34 \%$ had an ejection fraction over $50 \%$. Mean admission creatinine was $1.7 \pm 1.6 \mathrm{mg} / \mathrm{dl}$ and $19 \%$ had a creatinine over $2 \mathrm{mg} / \mathrm{dl}$. Serum creatinine increased more than $0.5 \mathrm{mg} / \mathrm{dl}$ during hospitalization in $9 \%$ of general patients and in $11 \%$ of diabetics. The increase in creatinine was associated with a higher risk of hospital mortality (odds ratio $(O R)$ 12.9, 95\% confidence intervals (CI) 6.7-27.6) and long term mortality (OR 2.1, 95\% CI 1.6-3). Conclusions: The deterioration of renal function during hospitalization of patients with heart failure is a risk factor for hospital and long term mortality.

(Rev Med Chile 2013; 141: 995-1002).

Key words: Heart failure; Renal insufficiency; Risk factors.

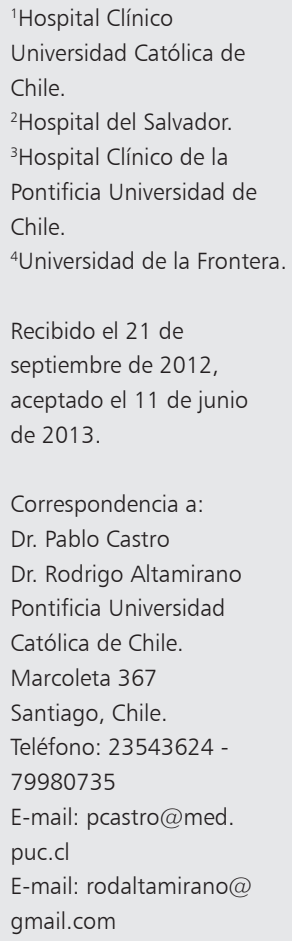

L a insuficiencia renal es un hallazgo frecuente en pacientes con insuficiencia cardiaca (IC) constituye un factor de pronóstico independiente de eventos adversos ${ }^{1-8}$. En distintas series más de la mitad de los pacientes que se hospitalizan por IC tienen deterioro de la función renal. En el año 2002 se creó el Registro Nacional de IC (grupo ICARO), que incluye a pacientes hospitalizados por IC en centros chilenos. Desde entonces se han estudiado las características clínicas, trata- miento, evolución intrahospitalaria y alejada de estos pacientes. En un primer análisis que incluyó 646 pacientes se encontró que la edad avanzada, insuficiencia renal, y el manejo en un centro no metropolitano identifican un grupo de alto riesgo de mortalidad en el seguimiento alejado. (Abstract Revista Chilena de Cardiología 2008).

Series internacionales han mostrado que durante la hospitalización puede existir un mayor deterioro de la función renal. Esto ocurre en 
alrededor de $30-50 \%$ de los pacientes hospitalizados. Esto se ha asociado a eventos adversos como hospitalización prolongada, mortalidad intrahospitalaria y alejada y mayores tasas de rehospitalización. Sin embargo, estas observaciones se han obtenido en series que incluyen un número relativamente pequeño de pacientes, muchas de ellas restringidas a pacientes mayores de 65 años $^{9-}$ ${ }^{12} \mathrm{y}$ limitadas al seguimiento intrahospitalario o a un período breve post alta ${ }^{9-13-14}$. Por otro lado, por ser estudios con un relativo bajo número de pacientes se desconocen los factores asociados o predisponentes a deterioro de la función renal. El impacto del deterioro de la función renal en la sobrevida alejada de pacientes hospitalizados a nivel nacional no ha sido evaluado.

El objetivo del presente estudio es determinar la prevalencia del deterioro de la función renal, sus determinantes y la relación con la mortalidad alejada en pacientes ingresados con diagnóstico de IC a hospitales chilenos pertenecientes al registro ICARO.

\section{Métodos}

Desde enero de 2002 a julio de 2009, en 14 centros chilenos, se recolectó información prospectiva de 1.164 pacientes hospitalizados por IC en CF III o IV mediante una ficha electrónica específicamente diseñada. El criterio diagnóstico fue: síntomas de IC y evidencias objetivas de disfunción cardiaca en el ecocardiograma o la radiografía de tórax; en casos dudosos se consideró la respuesta al tratamiento para $\mathrm{IC}^{15}$. Se excluyeron todos los pacientes en los cuales el motivo principal de hospitalización no fue IC y aquellos pacientes previamente asintomáticos y sin antecedentes de IC, que presentaron IC o shock cardiogénico secundario a un síndrome coronario agudo. Se registraron características demográficas, comorbilidades, previsión, nivel educacional, antecedentes de hospitalizaciones por IC, etiología de la cardiopatía de base, factores de descompensación actuales, hallazgos del electrocardiograma y ecocardiograma, incluida la fracción de eyección de ventrículo izquierdo (FE). Se consignó el tratamiento al ingreso, alta y evolución intrahospitalaria. Las historias clínicas, incluyendo radiografías de tórax, creatinina de ingreso y alta y ecocardiogramas fueron revisadas por el equipo investigador de cada centro. Los pacientes fueron categorizados en aquellos con creatinina de ingreso $<2 \mathrm{mg} / \mathrm{dl}$ y aquellos con creatinina $\geq 2 \mathrm{mg} / \mathrm{dl}$. En 441 pacientes se constató el peso al ingreso, en ellos se calculó la velocidad de filtración glomerular (VFG) de acuerdo a la fórmula de Cockcroft-Gault $=(140$-edad $\mathrm{x}$ peso en $\mathrm{kg} / 72$ x creatinina plasmática en $\mathrm{mg} / \mathrm{dl}$ ) x 0,85 en caso de sexo femenino ${ }^{16}$.

Se definió deterioro de la función renal como un incremento en la creatinina plasmática mayor a $0,5 \mathrm{mg} / \mathrm{dl}$ durante la hospitalización. En caso de contar con la estimación de la VFG se definió deterioro renal como una disminución $\geq 25 \%$ de la VFG. La mortalidad al fin del seguimiento se determinó por la base de datos del Servicio Nacional de Registro Civil e Identificación.

\section{Análisis estadístico}

Los objetivos primarios del estudio fueron analizar las variables asociadas al deterioro de la función renal e investigar el valor pronóstico de éste, sobre la mortalidad alejada. Las características de la población son expresadas como media \pm desviación standard para variables continuas y como porcentaje (frecuencia) para variables categóricas. Las diferencia entre los grupos con deterioro o sin deterioro de la función renal fueron analizadas mediante T Student para muestras independientes (para las variables continuas) y $\chi^{2}$ o test exacto de Fisher, según correspondía, para las variables categóricas, con un límite de 0,05; (con dos colas) para significancia estadística. La prevalencia de deterioro de la función renal utilizando ambas definiciones (incremento de la creatinina o disminución de la VFG) se comparó mediante $\chi^{2}$. La sobrevida se evaluó mediante método de Kaplan-Meier. Los predictores de mortalidad intrahospitalaria y a largo plazo se evaluaron mediante regresión logística y modelo de riesgo proporcional de Cox, respectivamente. En la construcción de este modelo se seleccionaron las variables consideradas biológicamente relevantes (edad, hipotensión y/o shock al ingreso, $\mathrm{FE}<35 \%$, diabetes).

\section{Resultados}

\section{a) Características de los pacientes}

Se incorporaron 1.164 pacientes. De ellos 1.100 contaban con determinaciones de creatinina. Cuarenta y cuatro coma nueve por ciento de los individuos era de sexo femenino; la edad promedio 
del grupo fue de 67,9 $\pm 13,3$ años. Del total de pacientes, 900 de ellos $(80,9 \%)$ tenían creatinina de ingreso $<2 \mathrm{mg} / \mathrm{dl}$ y 200 pacientes $(19,1 \%)$ creatinina $\geq 2 \mathrm{mg} / \mathrm{dl}$. En el grupo total, $9,6 \%$ de los pacientes presentó un incremento de la creatinina $>0,5 \mathrm{mg} / \mathrm{dl}$. En el grupo con creatinina de ingreso menor a $2 \mathrm{mg} / \mathrm{dl}, 6,8 \%$ presentó deterioro de la función renal. En la Tabla 1 se muestran las características de los pacientes con creatinina al ingreso $<2 \mathrm{mg} / \mathrm{dl}$ de acuerdo a si presentaron (o no) deterioro de la función renal (definida por incremento mayor a $0,5 \mathrm{mg} / \mathrm{dl}$ ).
La etiología de IC más prevalente en la cohorte fue la hipertensiva $(39,3 \%)$ seguida por enfermedad coronaria (24,9\%). 34,4\% de la población se encontraba en CF III-IV previo a la descompensación. La hipertensión arterial y la diabetes fueron las comorbilidades más frecuentes (80\% y 40\% respectivamente). La presentación clínica más frecuente fue congestión pulmonar, presentándose en cerca de $90 \%$ de los pacientes al momento del ingreso. Tres por ciento de la población se presentó en shock cardiogénico.

Al comparar los grupos con y sin deterioro de

Tabla 1. Características basales de los pacientes con creatinina basal $<2 \mathbf{~ m g} / \mathbf{d L}$, con y sin deterioro función renal

\begin{tabular}{|c|c|c|c|}
\hline & Sin & Con & $\mathbf{p}$ \\
\hline Edad, media (DS), años & $67,64 \pm 13,72$ & $67,55 \pm 14,01$ & 0,96 \\
\hline Sexo Masculino, (\%) & 60 & 58 & 0,86 \\
\hline $\begin{array}{l}\text { Etiología, n (\%) } \\
\text { Hipertensiva } \\
\text { Isquémica } \\
\text { Dilatada }\end{array}$ & $\begin{array}{l}42,1 \\
25 \\
18\end{array}$ & $\begin{array}{l}39 \\
23 \\
17\end{array}$ & 0,82 \\
\hline $\begin{array}{l}\text { CF previa a descompensación. NYHA, (\%) } \\
\text { III } \\
\text { IV }\end{array}$ & $\begin{array}{r}31 \\
4\end{array}$ & $\begin{array}{r}30 \\
5\end{array}$ & 0,78 \\
\hline $\begin{array}{l}\text { Comorbilidades, n (\%) antecedentes } \\
\text { HTA } \\
\text { DM } \\
\text { Infarto previo } \\
\text { EPOC } \\
\text { AVE } \\
\text { FA }\end{array}$ & $\begin{array}{r}524(80) \\
223(37) \\
135(23) \\
83(14) \\
65(11) \\
182(35)\end{array}$ & $\begin{array}{r}55(80) \\
29(43) \\
14(22) \\
8(13) \\
9(14) \\
17(27)\end{array}$ & $\begin{array}{l}1 \\
0,43 \\
1 \\
0,85 \\
0,54 \\
0,21\end{array}$ \\
\hline $\begin{array}{l}\text { Forma de Presentación, (\%) } \\
\text { Hipotensión y/o shock } \\
\text { Congestión pulmonar }\end{array}$ & $\begin{array}{l}8,1 \\
91\end{array}$ & $\begin{array}{l}22 \\
93\end{array}$ & $p<0,01$ \\
\hline FEVI (\%) & $39,32+16,8$ & $41,46+20,2$ & 0,45 \\
\hline $\begin{array}{l}\text { Terapia al ingreso, n (\%) } \\
\text { IECA } \\
\text { ARA II } \\
\text { Betabloqueadores } \\
\text { Hidralazina } \\
\text { Nitratos } \\
\text { Digoxina } \\
\text { Furosemida } \\
\text { Espironolactona } \\
\text { Amiodarona } \\
\text { Bloq calcio } \\
\text { Anticoagulante oral } \\
\text { Aspirina }\end{array}$ & $\begin{array}{r}307(45) \\
72(11) \\
222(27) \\
43(6) \\
127(19) \\
148(22) \\
378(55) \\
151(22) \\
71(10) \\
95(14) \\
199(29) \\
274(40)\end{array}$ & $\begin{array}{r}26(38) \\
3(4) \\
19(32) \\
6(9) \\
14(20) \\
15(22) \\
37(54) \\
15(22) \\
10(15) \\
11(16) \\
22(32) \\
29(42)\end{array}$ & $\begin{array}{c}0,26 \\
0,14 \\
0,49 \\
0.44 \\
0,75 \\
1 \\
0,8 \\
1 \\
0,31 \\
0,6 \\
0,68 \\
0,8\end{array}$ \\
\hline
\end{tabular}


la función renal se observó que la distribución por género, $\mathrm{CF}$ al ingreso y antecedentes fueron similares, con una tendencia no significativa a mayor proporción de diabéticos en el subgrupo de pacientes con deterioro de la función renal $(11,5 \%$ vs $9,3 \%, p=0,21)$. La presentación clínica fue mayoritariamente congestión pulmonar en ambos grupos siendo la hipotensión o shock más frecuente en los pacientes con deterioro renal $(22,6 \%$ vs $8,1 \%, \mathrm{p}<0,01)$, sin existir diferencias en relación a la terapia médica de ingreso. Tampoco hubo diferencias en la fracción de eyección ventricular izquierda, siendo en promedio de $39,32 \pm 16,8 \%$ vs $41,46 \pm 20,2 \%(p=0,4)$. No hubo diferencias significativas en los registros de presión arterial.

En relación a la terapia intrahospitalaria los pacientes con deterioro de la función renal requirieron más inótropos, monitoreo hemodinámico invasivo ventilación mecánica y diálisis (Tabla 2).

En 441 pacientes (40\%) se pudo calcular la VFG por la fórmula de Cockcroft-Gault (64 \pm $35,8 \mathrm{ml} / \mathrm{min}$ para la población en estudio). En este grupo la incidencia de deterioro de la función renal estimada por una disminución de la VFG $\geq 25 \%$ fue similar a cuando se utilizó el incremento creatinina $>0,5 \mathrm{mg} / \mathrm{dl}(12,6$ vs $9,2 \%$, en el grupo total, $\mathrm{p}=0,12)$.

\section{b) Terapia farmacológica la ingreso y al alta}

Los datos de la terapia al ingreso de los pacientes con creatinina basal $<2 \mathrm{mg} / \mathrm{dl}$ se muestran en la Tabla 1. Se observa que no existen diferencias significativas en las terapias farmacológicas entre los pacientes que presentaron o no deterioro de la función renal. Sin embargo, la terapia al egreso hospitalario (Tabla 2) difiere significativamente. En los pacientes con deterioro de la función renal existió un menor uso de inhibidores de la enzima convertidora de angiotensina o antagonistas de los receptores de angiotensina II (30\% vs $66 \%$, $\mathrm{p}<0,01)$, menor uso de betabloqueadores $(30 \%$ vs $44 \%, \mathrm{p}=0,03)$ y menor utilización de espironolactona ( $26 \%$ vs $47 \%), \mathrm{p}<0,01)$. Medicamentos como la hidralazina y nitratos se utilizaron en una proporción similar.

\section{c) Evolución intrahospitalaria y alejada}

La mortalidad intrahospitalaria fue significativamente mayor en aquellos pacientes con deterioro de la función renal durante la hospitalización (31,9 vs 3,5\%, p < 0,01, OR 12,87 IC95\% 6,72$24,65)$. El seguimiento promedio de la cohorte fue de $635 \pm 581$ días post alta, con una sobrevida de $70,4 \pm 1,4 \%$ en el primer año.

En la Figura 1 se muestran las curvas de sobrevida en pacientes con y sin deterioro de la

Tabla 2. Terapia intrahospitalaria y al egreso de los pacientes con creatinina basal $<2$ mg/dl, según ausencia o deterioro de la función renal

\begin{tabular}{|c|c|c|c|}
\hline & Sin deterioro & Con deterioro & $\mathbf{p}$ \\
\hline $\begin{array}{l}\text { Intrahospitalaria, n (\%) } \\
\text { Inótropos } \\
\text { Vasodilatadores } \\
\text { Diálisis } \\
\text { Swan Ganz } \\
\text { Ventilación mecánica }\end{array}$ & $\begin{array}{c}165(29) \\
233(40) \\
19(3) \\
22(4) \\
54(10)\end{array}$ & $\begin{array}{l}30(47) \\
22(34) \\
11(17) \\
10(16) \\
13(21)\end{array}$ & $\begin{array}{c}<0,01 \\
0,42 \\
<0,01 \\
<0,01 \\
0,02\end{array}$ \\
\hline $\begin{array}{l}\text { Terapia farmacológica al alta } \\
\text { IECAy/oARAIl } \\
\text { Betabloqueadores } \\
\text { Hidralazina } \\
\text { Nitratos } \\
\text { Digoxina } \\
\text { Furosemida } \\
\text { Espironolactona } \\
\text { Amiodarona } \\
\text { Bloq calcio } \\
\text { Anticoagulante oral } \\
\text { Aspirina }\end{array}$ & $\begin{array}{c}448(66) \\
303(44) \\
99(15) \\
132(19) \\
187(27) \\
514(75) \\
320(47) \\
118(17) \\
73(11) \\
253(37) \\
316(42)\end{array}$ & $\begin{array}{c}21(30) \\
21(30) \\
15(22) \\
17(25) \\
8(12) \\
43(62) \\
18(26) \\
13(19) \\
10(15) \\
19(28) \\
23(33)\end{array}$ & $\begin{array}{c}<0,01 \\
0,03 \\
0,11 \\
0,34 \\
<0,01 \\
0,03 \\
<0,01 \\
0,74 \\
0,32 \\
0,15 \\
0,04\end{array}$ \\
\hline
\end{tabular}



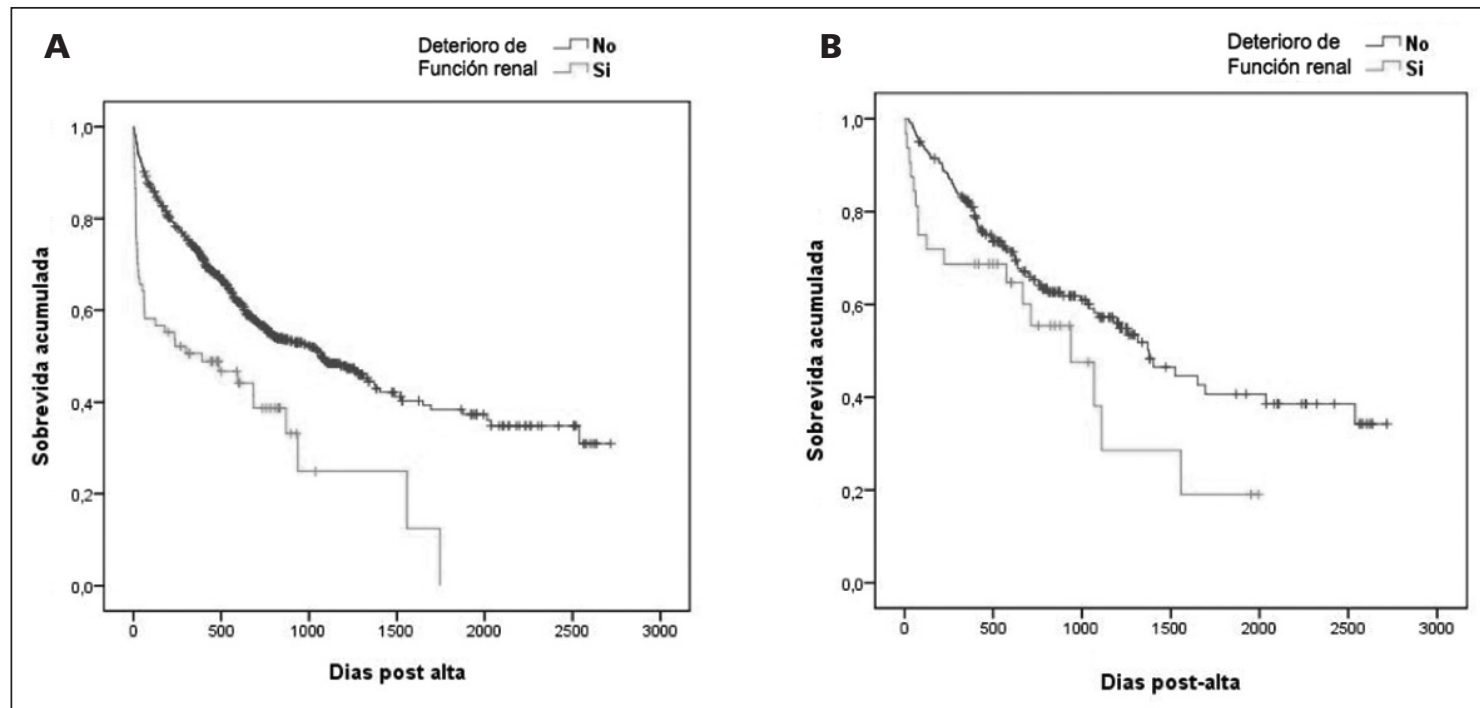

Figura 1. Curvas de sobrevida según deterioro de la función renal. A: incremento creatinina $>0,5 \mathrm{mg} / \mathrm{dl}$. B: disminución VFG $\geq 25 \%$.

función renal de acuerdo a ambas definiciones. La mortalidad alejada fue significativamente mayor en los pacientes con deterioro de la función renal independiente del criterio utilizado.

En la Figura 2 se muestran los resultados del análisis multivariado. Se observa que en los mayores

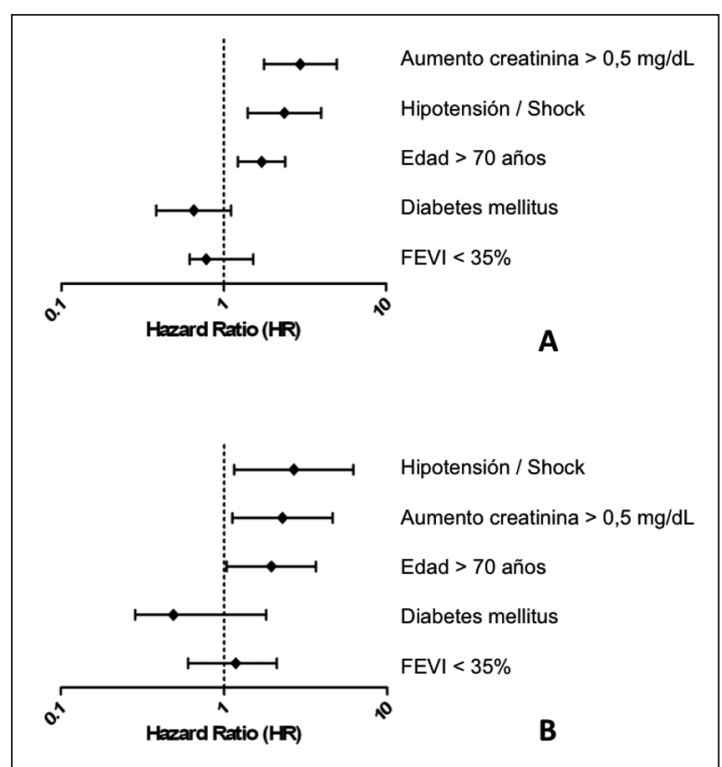

Figura 2. Análisis multivariado de predictores de mortalidad. A: se incluyen a todos los pacientes. B: pacientes con creatinina basal $<2 \mathrm{mg} / \mathrm{dl}$. de 70 años, la presencia de hipotensión y/o shock al ingreso y el deterioro de la función renal fueron predictores independientes de mortalidad en el grupo global (HR 2,14, IC95\% 1,55-2,96) y en el grupo con creatinina basal $<2 \mathrm{mg} / \mathrm{dl}$. La presencia de diabetes no alcanzó significancia estadística. En la Figura 3 se muestra el riesgo de mortalidad alejada, relacionada a distintos grados de incremento de la creatinina plasmática durante la hospitalización

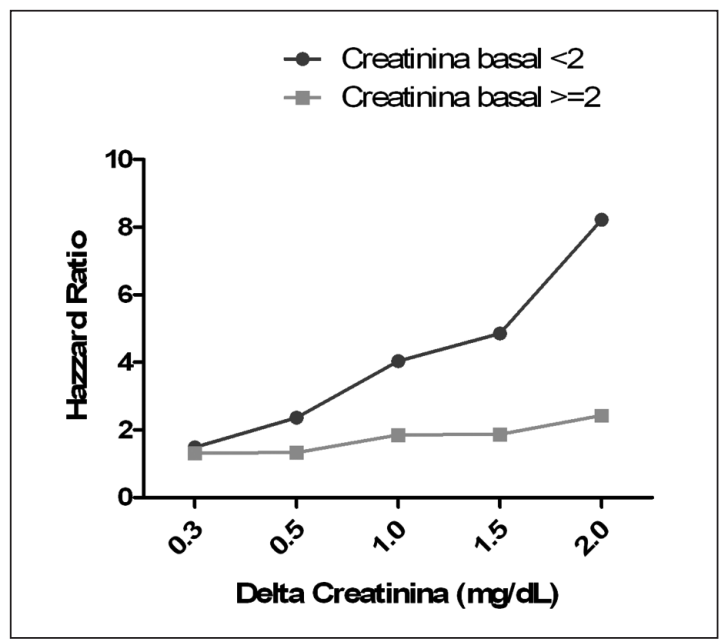

Figura 3. Valor pronóstico de la magnitud del incremento de la creatinina en la población de pacientes de acuerdo a la creatinina basal. 
tanto en pacientes con creatinina de ingreso $\geq 2$ $\mathrm{mg} / \mathrm{dl} \mathrm{y}<2 \mathrm{mg} / \mathrm{dl}$. Se observa que el riesgo de mortalidad alejada se manifiesta con incrementos de $0,5 \mathrm{mg} / \mathrm{dl}$ o más. Su impacto pronóstico es significativamente mayor en pacientes con creatinina basal $<2 \mathrm{mg} / \mathrm{dl}$ ( $\mathrm{p}<0,01$ para la comparación).

\section{Discusión}

La función renal es un determinante pronóstico reconocido en la IC. En pacientes hospitalizados por IC descompensada existe una estimulación neurohumoral aumentada, fenómenos de vasoconstricción renal e hipoperfusión que pueden deteriorar aun más la función renal. Sin embargo, la prevalencia de este hallazgo y su rol pronóstico no ha sido estudiada en grandes series de pacientes representativas del mundo real. Además de las características de la población (comorbilidades, edad, inclusión de pacientes con FE preservada o terapias), existen amplias variaciones en la definición de deterioro de la función renal. Algunos autores han utilizado cambios en los niveles de creatini$\mathrm{na}^{17-19}, \mathrm{VFG}^{20}$, o en valores de nitrógeno ureico ${ }^{21}$.

El deterioro de la función renal ocurrió en $9,2 \%$ de los pacientes hospitalizados por IC y en $6,8 \%$ de los pacientes con creatinina basal $<2 \mathrm{mg} /$ dl. Debido a que desde el punto de vista teórico a menores niveles de creatinina un incremento dado afecta en forma inversa y exponencial los valores de filtración glomerular ${ }^{22}$ decidimos comparar las incidencias obtenidas con ambas definiciones (deterioro de VFG estimado $\geq 25 \%$ vs ascenso creatinina plasmática $>0,5 \mathrm{mg} / \mathrm{dl}$ ), sin encontrarse diferencias significativas según el empleo de una u otra definición. En comparación con otros estudios, observamos una menor incidencia de deterioro de la función renal ${ }^{22}$. Esto podría deberse a la inclusión de pacientes con un rango de edad más amplio, FE conservada y menor proporción de pacientes con falla renal previa ${ }^{23}$. Adicionalmente, pueden existir diferencias en relación a la severidad de la IC o a las terapias utilizadas.

Con respecto a la severidad de la IC estimada indirectamente por el requerimiento de inótropos no se observan diferencias con otros estudios ${ }^{24}$.

Mayores incrementos de creatinina durante la hospitalización confieren un pronóstico más desfavorable especialmente en aquellos pacientes con creatinina plasmática basal $<2 \mathrm{mg} / \mathrm{dl}$.

El deterioro de la función renal se asoció a presencia de hipotensión y/o shock, mayor empleo de inótropos, ventilación mecánica y necesidad de diálisis (se excluyeron pacientes en diálisis crónica), lo que puede estar en concomitancia con mayor gravedad de la IC. En relación a comorbilidades, existió una tendencia a mayor prevalencia de diabetes mellitus, sin embargo, no fue significativa.

Distintos factores en la literatura se han asociado a riesgo de síndrome cardiorenal. Este síndrome ha tenido definiciones variables ${ }^{25-27}$, pero en general se considera un estado en el que existe una alteración de la interacción cardio-renal manifestada por uno o más de de tres hechos específicos. Ellos incluyen IC con enfermedad renal significativa concomitante, deterioro de la función renal durante el tratamiento de la descompensación y resistencia a diuréticos. Los factores de riesgo comunes como hipertensión arterial, diabetes mellitus y ateroesclerosis explican la alta coexistencia de disfunción cardiaca y renal ${ }^{28}$. En general los pacientes que desarrollan falla renal son de edad más avanzada, tienen mayor prevalencia de IC, falla renal previa, hipertensión y diabetes ${ }^{25-27}$.

En la IC aguda, el deterioro de la función renal parece ser más acentuado en aquellos pacientes con mayor deterioro de la función sistólica del VI, que en aquellos con FE preservada, alcanzando una incidencia de hasta $70 \%$ en los pacientes en shock cardiogénico $^{29-30}$. No se encontraron diferencias en la incidencia de falla renal en pacientes con FE preservada o disminuida. Esto está limitado por el número de pacientes en los que se dispone de esta información $(63 \%)$ y por la escasez de pacientes con deterioro marcado de la FE.

La falla renal en relación al tratamiento de la IC es manifestación de la disminución de la perfusión renal secundaria a la depleción de volumen o bien a una caída del gasto cardiaco pese a hipervolemia. Es interesante que la falla renal ocurre precozmente en el tratamiento de la descompensación cardiaca, cuando los pacientes aún se encuentran congestivos ${ }^{31}$. También debe ser considerada la velocidad de depleción dado que existe un desplazamiento de volúmenes entre el intersticio y el intravascular que está retrasado con respecto a la diuresis ${ }^{32,33}$.

\section{Importancia pronóstica del deterioro de la función renal}

En nuestra serie de pacientes el deterioro de la función renal fue un predictor independiente de 
mortalidad intrahospitalaria y alejada. El deterioro de la función renal se ha asociado a un pronóstico

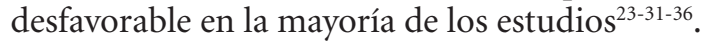

La incidencia variable de deterioro de la función renal se debe en parte a variaciones en las definiciones utilizadas. Se ha planteado la utilización exclusiva del cambio en la creatinina y no la $\mathrm{VFG}^{24}$. Es posible que parte del impacto desfavorable del deterioro de la función renal este dado por que la falla renal y en algunos casos la hiperkalemia puede limitar la utilización de terapias de reconocido efecto benéfico en la sobrevida de pacientes con IC (inhibidores de la enzima convertidora de angiotensina, antagonistas de los receptores de angiotensina II o espironolactona).

En el grupo con deterioro de la función renal, se observó una menor indicación de estos fármacos al alta, esto no se acompañó de un aumento en la indicación de hidralazina o nitratos.

En cuanto al deterioro de la función renal en relación a los valores de creatinina basal, se observó que el riesgo de mortalidad es mayor para los pacientes con creatinina basal menor a $2 \mathrm{mg} / \mathrm{dl}$.

Dentro de las limitaciones de este estudio se encuentran las limitaciones inherentes a la definición de deterioro renal. La ausencia de datos con respecto a la diuresis, lo que limita la utilización de sistemas más actualizados de insuficiencia renal. No se realizaron determinaciones de FE en todos los pacientes y se desconoce la adherencia al tratamiento en el seguimiento alejado, como también la evolución de la función renal.

\section{Conclusión}

El principal hallazgo de este estudio es que el deterioro de la función renal es un predictor independiente de mortalidad intrahospitalaria y en el seguimiento alejado, incluso en pacientes con creatinina basal menor a $2 \mathrm{mg} / \mathrm{dl}$.

La incidencia de falla renal, se asoció a una menor utilización de terapias de reconocida eficacia para el manejo de la IC.

\section{Referencias}

1. Smith GL, Lichtman JH, Bracken MB, Shlipak MG, Phillips CO, DiCapua P. Renal impairment and outcomes in heart failure: systematic review and meta-analysis. J Am Coll Cardiol 2006; 47: 1987-96.
2. McAlister FA, Ezekowitz J, Tonelli M, Armstrong PW. Renal insufficiency and heart failure: prognostic and therapeutic implications from a prospective cohort study. Circulation 2004; 109: 1004-9.

3. Hillege HL, Girbes AR, de Kam PJ, Boomsma F, de Zeeuw D, Charlesworth A, et al. Renal function, neurohormonal activation, and survival in patients with chronic heart failure. Circulation 2000; 102: 203-10.

4. De Silva R, Nikitin NP, Witte KK, Rigby AS, Goode K, Bhandari S, et al. Incidence of renal dysfunction over 6 months in patients with chronic heart failure due to left ventricular systolic dysfunction: contributing factors and relationship to prognosis. Eur Heart J 2006; 27: 569-81.

5. Shlipak MG, Smith GL, Rathore SS, Massie BM, Krumholz HM. Renal function, digoxin therapy, and heart failure outcomes: evidence from the digoxin intervention group trial. J Am Soc Nephrol 2004; 15: 2195-203.

6. Dries DL, Exner DV, Domanski MJ, Greenberg B, Stevenson LW. The prognostic implications of renal insufficiency asymptomatic and symptomatic patients with left ventricular systolic dysfunction. J Am Coll Cardiol 2000; 35: 681-9.

7. Shlipak MG, Massie BM. The clinical challenge of cardiorenal syndrome. Circulation 2004; 110: 1514-7.

8. Mahon NG, Blackstone EH, Francis GS, Starling III RC, Young JB, Lauer MS. The prognostic value of estimated creatinine clearance alongside functional capacity in ambulatory patients with chronic congestive heart failure. J Am Coll Cardiol 2002; 40 (6): 1106-13.

9. Smith GL, Vaccarino V, Kosiborod M, Lichtman JH, Cheng S, Watnick SG, et al. Worsening renal function: what is a clinically meaningful change in creatinine during hospitalization with heart failure? J Card Fail 2003; 9: 13-25.

10. Cowie MR, Komajda M, Murray-Thomas T, Underwood J, Ticho B. Prevalence and impact of worsening renal function in patients hospitalized with decompensated heart failure: results of the prospective outcomes study in heart failure (POSH). Eur Heart J 2006; 27: 1216-22.

11. Akhter MW, Aronson D, Bitar F, Khan S, Singh H, Singh $\mathrm{RP}$, et al. Effect of elevated admission serum creatinine and its worsening on outcome in hospitalized patients with decompensated heart failure. Am J Cardiol 2004; 94: 957-60.

12. Forman DE, Butler J, Wang Y, Abrahan WT, O'Connor $\mathrm{CM}$, Gottlieb S, et al. Incidence, predictors at admission, and impact of worsening renal function among patients hospitalized with heart failure. J Am Coll Cardiol 2004; 43: 61-7.

13. Krumholz HM, Chen YT, Vaccarino V, Wang Y, Radford 
MJ, Bradford WD, et al. Correlates and impact on outcomes of worsening renal function in patients $\mathrm{N}$ or $=65$ years of age with heart failure. Am J Cardiol 2000; 85: 1110-3.

14. Gottlieb SS, Abraham W, Butler J, Forman DE, Loh E, Massie BM, et al. The prognostic importance of different definitions of worsening renal function in congestive heart failure. J Card Fail 2002; 8: 136-41.

15. Castro P, Vukasovic JL, Garcés E, Sepúlveda L, Ferrada M, Alvarado S. Insuficiencia Cardiaca: Registro y Organización. Insuficiencia cardiaca en hospitales chilenos: resultados del registro nacional de insuficiencia cardiaca. ICARO 2004; 132 (6): 655-62.

16. Cockcroft DW, Gault MH. Prediction of creatinine clearance from serum creatinine. Nephron 1976; 16: 31-41.

17. Forman DE, Butler J, Wang Y, Abraham WT, O'Connor $\mathrm{CM}$, Gottlieb SS, et al. Incidence, predictors at admission, and impact of worsening renal function among patients hospitalized with heart failure. J Am Coll Cardiol 2004; 43: 61-7.

18. Pfister R, Diedrichs H, Schiedermair A, Rosenkranz S, Hellmich M, Erdmann E, et al. Prognostic impact of NT-proBNP and renal function in comparison to contemporary multi-marker risk scores in heart failure patients. Eur J Heart Fail 2008; 10 (3): 315-20.

19. Pitt B, Segal R, Martínez FA, Meurers G, Cowley AJ, Thomas I, et al. Randomised trial of losartan versus captopril in patients over 65 with heart failure (Evaluation of Losartan in the Elderly Study, ELITE). Lancet 1997; 349: 747-52.

20. Stevens LA, Coresh J, Greene T, Levey AS. Assessing kidney function-measured and estimated glomerular filtration rate. N Engl J Med 2006; 354: 2473-83.

21. Cauthen CA, Lipinski MJ, Abbate A, Appleton D, Nusca A, Varma A, et al. Relation of blood urea nitrogen to long-term mortality in patients with heart failure. Am J Cardiol 2008; 1; 101 (11): 1643-

22. Klein L, Massie BM, Leimberger JD, O'Connor CM, Piña IL, Adams KF Jr, et al. OPTIME-CHF Investigators. Admission or changes in renal function during hospitalization for worsening heart failure predict postdischarge survival: results from the Outcomes of a Prospective Trial of Intravenous Milrinone for Exacerbations of Chronic Heart Failure (OPTIME-CHF). Circ Heart Fail 20081 (1): 25-33.

23. Damman K, Navis G, Voors AA, Asselbergs FW, Smilde TD, Cleland JG, et al. Worsening renal function and prognosis in heart failure: systematic review and metaanalysis. J Card Fail 2007; 13 (8): 599-608.

24. Metra M, Nodari S, Parrinello G, Bordonali T, Bugatti $\mathrm{S}$, Danesi R, et al. Worsening renal function in patients hospitalised for acute heart failure: clinical implications and prognostic significance. Eur J Heart Fail 2008; 10 (2): 188-95.

25. Liang KV, Williams AW, Greene EL, Redfield MM. Acute decompensated heart failure and the cardiorenal syndrome. Crit Care Med 2008; 36 (1 Suppl): S75-88.

26. Zannad F, Adamopoulos C, Mebazaa A, Gheorghiade $\mathrm{M}$. The challenge of acute decompensated heart failure. Heart Fail Rev 2006; 11 (2): 135-9.

27. Francis G. Acute decompensated heart failure: the cardiorenal syndrome. Cleve Clin J Med 2006; 73 Suppl 2: S8-13.

28. Mehrotra R, Kathuria P. Place of peritoneal dialysis in the management of treatmentresistant congestive heart failure. Kidney Int Suppl 2006; (103): S67-S71.

29. Ronco C, Haapio M, House AA, Anavekar N, Bellomo R. Cardiorenal syndrome. J Am Coll Cardiol 2008; 52 (19): 1527-39.

30. Jose P, Skali H, Anavekar N, Tomson C, Krumholz HM, Rouleau JL, et al. Increase in creatinine and cardiovascular risk in patients with systolic dysfunction after myocardial infarction. J Am Soc Nephrol 2006; 17: 2886 -91.

31. Gottlieb SS, Abraham W, Butler J, Forman DE, Loh E, Massie BM, et al. The prognostic importance of different definitions of worsening renal function in congestive heart failure. J Card Fail 2002; 8: 136-41.

32. Firth JD, Raine AE, Ledingham JG. Raised venous pressure: A direct cause of renalsodium retention in oedema? Lancet 1988; 1: 1033-5.

33. Howard PA, Dunn MI. Aggressive diuresis for severe heart failure in the elderly. Chest 2001; 119: 807-10.

34. Gottlieb SS, Brater DC, Thomas I, Havranek E, Bourge R, Goldman S, et al. BG9719 (CVT-124), an A1 adenosine receptor antagonist, protects against the decline in renal function observed with diuretic therapy. Circulation 2002; 105: 1348-53.

35. Bart BA, Teerlink JR, Costanzo MR, Saltzberg M, Sobotka P. Changes in serum creatinine during treatment of heart failure and volume overload with ultrafiltration or intravenous diuretics. J Cardiac Fail 2006; 12 (6 Suppl): S114.

36. Ronco F, Ronco C. Cardiorenal syndrome, current understanding. Recenti Prog Med 2009; 100 (4): 202-13. 